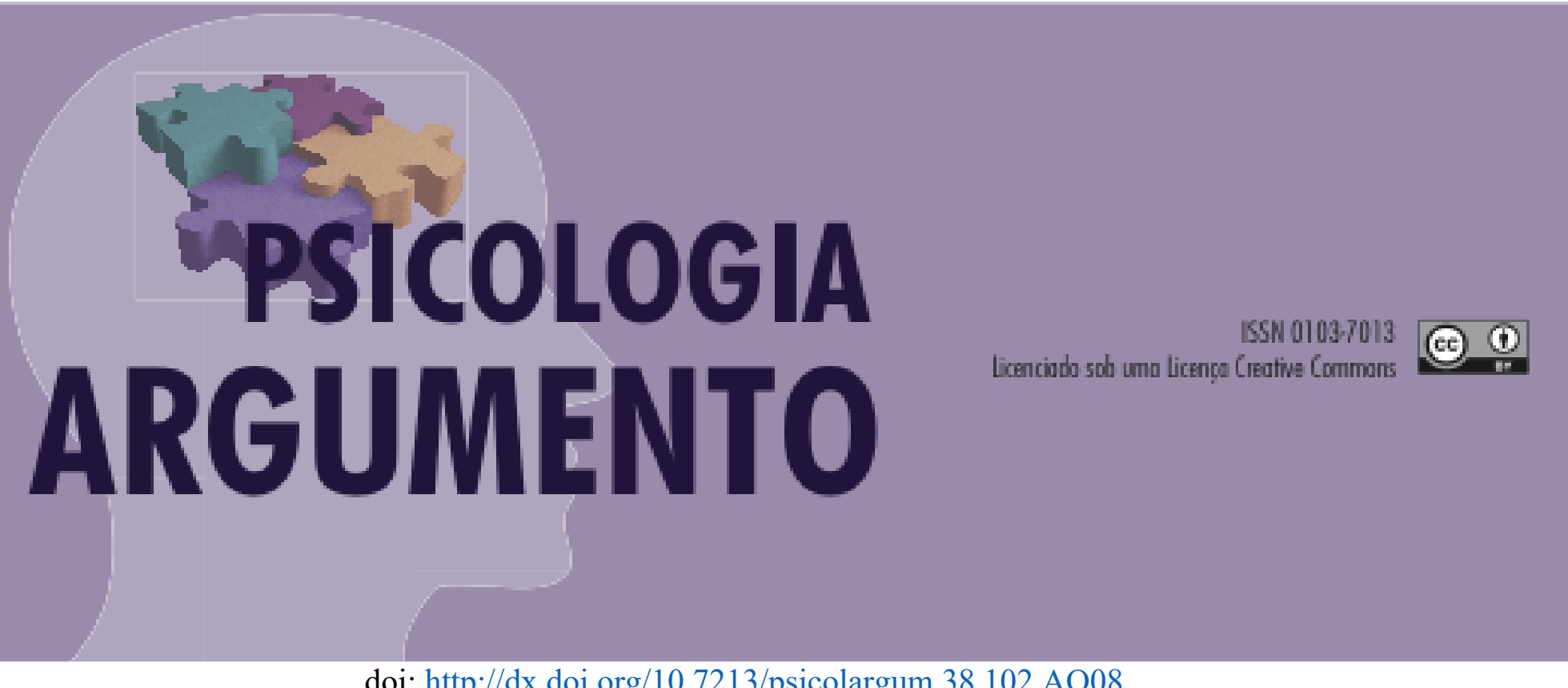

doi: http://dx.doi.org/10.7213/psicolargum.38.102.AO08

\title{
Revisão integrativa sobre a atenção da psicologia às gestantes surdas
}

Integrative review on the attention of psychology to deaf pregnant women

Revisión integradora de la atención psicológica para mujeres sordas embarazadas

Alexsandra Machado Maffei

Doutoranda em Desenvolvimento e Perturbações da Linguagem, Universidade

Fernando Pessoa, Porto, Portugal. Email: alexsandramaffei@gmail.com ou 14919@ufp.edu.pt

ORCID https://orcid.org/0000-0002-2180-1574

Rute Flávia Meneses Mondim Pereira d'Amaral

Professora Associada, FCHS/CTEC/OLD/APASD/CPP/FB-B2S - Universidade

Fernando Pessoa, Porto, Portugal. Email: rmeneses@ufp.edu.pt ORCID http://orcid.org/0000-

0002-7189-3139

\begin{abstract}
Resumo
A gestação é um período permeado por sentimentos/pensamentos ambivalentes pelos quais a Psicologia demonstra interesse. Os aspectos físicos/biológicos da gestação sempre foram temas centrais nos cuidados pré e pós-natais. Atualmente, programas de cuidados pré-natais psicológicos oferecem espaço acolhedor com o objetivo, por exemplo, de promover a saúde das gestantes e prevenir depressão pós-parto. É possível incluir, também, os companheiros(as) e a família estendida num ambiente de diálogo sobre crenças, expectativas, riscos e anseios presentes nesta fase. Numa breve revisão da bibliografia constata-se que a Psicologia dedica especial atenção aos cuidados das gestantes. O objetivo deste estudo foi verificar, na literatura, se as gestantes surdas têm
\end{abstract}


recebido esta mesma atenção. Realizou-se uma revisão integrativa da literatura, até o dia 31 de março de 2020. Foram consultadas as bases de dados PubMed, SciELO, EBSCO e BVS, sem uso de filtros tampouco limite quanto a data de publicação. Os termos MeSH/descritores utilizados foram pregnant women AND Psychology AND deaf*. Dentre os 52 artigos identificados, apenas nove foram incluídos neste estudo, sendo que oito deles não tinham como foco principal gestantes surdas e sim gestantes com deficiência. Gestantes com deficiência/surdez parecem, assim, excluídas tanto dos dados censitários, quanto dos serviços de saúde e das pesquisas científicas. Nessa amostra, apesar de pequena, depreendeu-se que estas mulheres são vítimas de preconceito e discriminação de diferentes instâncias da sociedade. No entanto, é possível constatar, também, que há um campo importante e necessário para a atuação da Psicologia voltado a essas mulheres, profissionais de saúde e familiares.

Palavras-chave: gravidez, Psicologia, surdez

\begin{abstract}
Pregnancy is a period permeated by ambivalent feelings/thoughts for which Psychology shows interest. The physical/biological aspects of pregnancy have always been central themes in pre- and post-natal care. Today, psychological prenatal care programs offer a welcoming space with the aim, for example, of promoting the health of pregnant women and preventing postpartum depression. It is also possible to include partners and extended family in an environment of dialogue about beliefs, expectations, risks and desires present in this phase. In a brief review of the literature, it can be seen that Psychology has devoted special attention to the care of pregnant women. The aim of this study was to verify, in the literature, whether deaf pregnant women have received this same attention. A integrative review of the literature was carried out until March 31, 2020. The PubMed, SciELO, EBSCO and VHL databases were consulted, and no filters were applied, nor a limit as to the date of publication. The MeSH terms/descriptors used were pregnant women AND Psychology AND deaf*. Among the 52 articles identified, only ninve were included in this study, and eight of them did not focus on deaf pregnant women, but disabled pregnant women. Therefore, pregnant women with disabilities/deafness seem to be excluded from census data, health services and scientific research. In this sample, although small, it was possible to infer that these women are victims of prejudice and discrimination from different levels of society. However, it is also possible to verify that there is an important and necessary field for the intervention of Psychology aiming to these women, health professionals and families.
\end{abstract}

Keywords: pregnancy, Psychology, deafness

\title{
Resumen
}

El embarazo es un período impregnado de sentimientos/pensamientos ambivalentes por los cuales la psicología muestra interés. Los aspectos fisicos/biológicos del embarazo siempre han sido temas centrales en la atención prenatal y postnatal. Hoy, los programas psicológicos de atención prenatal ofrecen un espacio acogedor con el objetivo, por ejemplo, de promover la salud de las mujeres embarazadas y prevenir la depresión posparto. También es posible incluir parejas y familiares en un ambiente de diálogo sobre creencias, expectativas, riesgos y deseos presentes en esta fase. En una breve revisión de la bibliografía, se puede ver que la psicología ha dedicado especial atención al cuidado de las mujeres embarazadas. El objetivo de este estudio fue verificar, en la literatura, si las mujeres embarazadas sordas han recibido esta misma 
atención. Se realizó una revisión integradora de la literatura hasta el 31 de marzo de 2020. Se consultaron las bases de datos PubMed, SciELO, EBSCO y BVS, sin el uso de filtros ni un límite en la fecha de publicación. Los términos/descriptores MeSH utilizados fueron mujeres embarazadas y psicología y sordos*. Entre los 52 articulos identificados, solo nueve se incluyeron en este estudio, de los cuales ocho no tenían mujeres embarazadas sordas como su foco principal, sino mujeres embarazadas con discapacidades. Por lo tanto, las mujeres embarazadas con discapacidad/sordera parecen estar excluidas tanto de los datos del censo, los servicios de salud y las investigaciones científicas. En esta muestra, aunque pequeña, fue posible inferir que estas mujeres son víctimas de prejuicios y discriminación de diferentes niveles de la sociedad. Sin embargo, también es posible notar que existe un campo importante y necesario para el trabajo de la Psicología dirigido a estas mujeres, profesionales de la salud y miembros de la familia.

Palabras clave: embarazo, psicología, sordera

\section{Introdução}

A gestação é marcada por inúmeras transformações, que se iniciam silenciosamente no corpo da mulher. As mudanças que eram apenas internas transbordam para o meio externo, provocando ações e reações em si e ao seu redor (Benincasa et al., 2019). Para além das mudanças corporais, essas mulheres vivem um conjunto de emoções intensas e contraditórias (Arrais \& Araújo, 2016) e algumas desconhecidas até o momento (Benincasa et al., 2019).

Programas que abordam as questões psíquicas nos cuidados pré-natais ganham espaço, num campo que antes era exclusivo dos aspectos físicos da gravidez. O objetivo destes é propiciar um espaço de escuta para a gestante e/ou familiares, uma vez que a gestação não é marcada "apenas" por sentimentos/pensamentos positivos (Almeida \& Arrais, 2016; Arrais \&Araujo, 2016; Benincasa et al., 2019).

Estes programas, coordenados por psicólogos, asseguram um ambiente acolhedor e sem julgamentos, com o propósito de promover a saúde dos participantes (Benincasa et al., 2019). A atenção à saúde psíquica da gestante, de seus(suas) companheiros(as) e da família estendida apresenta benefícios para um período permeado por ambivalências, crenças, expectativas e riscos (Almeida \& Arrais, 2016; Arrais \& Araujo, 2016; Benincasa et al., 2019). Constata-se, portanto, a relevância desta temática para a Psicologia e para a área da saúde. 
Infere-se que as gestantes surdas também compartilham dos sentimentos, pensamentos, condições físicas e emocionais das ouvintes, pelo que se justifica pensar em ações voltadas a este público. No entanto, para propô-los é preciso identificar essas mulheres e nem sempre é fácil acessar dados sobre as mesmas. Em Portugal, por exemplo, é possível aceder a informações censitárias a respeito do número de nascimentos/ano (PORDATA, 2019); no site do Instituto Nacional de Estatística (INE, 2019) obtem-se o número de mulheres surdas em idade fértil, não havendo um cruzamento de tais dados.

A despeito da saúde da população surda estudos indicam que esta quando comparada à população geral tende a sofrer mais abusos físicos e sexuais pelo parceiro(a) e tentar suicídio (Barnett et al., 2011), sofrer de depressão e abusar de álcool e tabaco (Woodcock \& Pole, 2007), apresentar sobrepeso e obesidade (Barnett et al., 2011; Mckee et al., 2011b) e dificuldade para obter informações necessárias à promoção de sua saúde (Anderson, Craig \& Ziedonis, 2017; Druel et al., 2018; Mckee et al., 2011a; Mckee et al., 2011b; Mckee et al., 2015; Woodcock \& Pole, 2007). Estes estudos sinalizam, portanto, a falta de acesso à informações, importantes e necessárias à promoção de saúde e prevenção de doenças de pessoas surdas.

As barreiras na comunicação entre profissionais e pessoas surdas tem sido responsável pela baixa procura aos serviços de saúde (Anderson et al., 2017; McKee et al., 2011a; Santos \& Portes, 2019) e por possíveis comprometimentos do diagnóstico ao tratamento (Marquete, Costa \& Teston, 2018; Reis \& Santos, 2019). Estresse e sentimentos negativos tais como, medo, insegurança e frustração, têm sido expressos por usuários surdos, nas suas experiências com o sistema de saúde (Santos \& Portes, 2019). Tais pesquisas evidenciam que a relação entre profissionais de saúde e esta população precisa receber uma atenção especial.

Estudos com gestantes surdas são ainda mais parcos, porém também revelam barreiras na comunicação destas com os profissionais de saúde. Um estudo comparativo entre gestantes ouvintes e surdas constatou que 91\% daquelas obtiveram muitas informações de seus médicos, enquanto $61 \%$ dessas referiram o mesmo (O’Hearn, 2006). Num hospital francês, equipes de saúde eram treinadas em língua gestual/sinais, atendiam a maioria das gestantes surdas, quando as consultas eram agendadas, no entanto, em situações de emergência menos de um quarto destas mulheres eram atendidas por estes profissionais (Equy et al., 2012). 
Wechsler, Reis e Ribeiro (2016) constataram que os níveis de depressão em gestantes reduziram em função da comunicação com os profissionais de saúde. Verifica-se, portanto, a importância dos cuidados pré-natais para a saúde manternoinfantil, com ampliação desses cuidados voltados à saúde mental da gestante/mãe/familiares (Almeida \& Arrais, 2016; Arrais \&Araujo, 2016; Benincasa et al., 2019). Logo o desafio deste estudo foi analisar a literatura sobre a atenção dada às gestantes surdas pela Psicologia.

\section{Método}

Realizou-se uma revisão integrativa da literatura indexada na PubMed, SciELO (Scientific Electronic Library Online), EBSCO e BVS (Biblioteca Virtual em Saúde), até o dia 31 de março de 2020. Os termos Medical Subject Headings $(\mathrm{MeSH}) /$ Descritores utilizados foram: pregnant women AND Psychology AND deaf*, sem limite quanto a data da publicação e sem filtros.

Ainda que se trate de uma revisão integrativa, teve-se em consideração o PRISMA (Preferred Reporting Items for Systematic Reviews and Meta-Analyses) Statement, observando as fases sugeridas pelo respectivo fluxograma, as quais consistem na identificação, passando pela seleção e elegibilidade até a inclusão de artigos para análise qualitativa (Moher, Tetzlaff \& The Prisma Group, 2009). Após a identificação dos estudos, foram removidos aqueles duplicados, assim como foram excluídos textos a partir: (a) da leitura dos títulos, (b) da leitura dos resumos, (c) da indisponibilidade dos artigos de forma gratuita. Apesar de haver dois revisores envolvidos, o processo não foi realizado na íntegra, de forma independente, por ambos.

Devido ao número bastante reduzido de estudos encontrados, o único critério de inclusão utilizado, nesta revisão, foi a atenção dada às gestantes surdas, independentemente desta condição ser a caracterização principal da gestante. Dito de outra maneira, aqueles estudos sobre gestantes com deficiência foram incluídos, porque dentre as participantes havia gestantes surdas.

A extração dos dados pautou-se na elaboração de um quadro, cujo objetivo foi sistematizar os conteúdos disponíveis nos artigos selecionados. Foram extraídas as seguintes informações: cuidados psicológicos pré/peri e pós-natais dirigidos às gestantes surdas/com deficiência, dificuldades/barreiras nos cuidados recebidos e formas de 
comunicação/apoio voltadas às gestantes com deficiência/surda. Todavia, face aos conteúdos extraídos, optou-se por não os apresentá-los em forma de quadro e sim em categorias temáticas, as quais são apresentadas e discutidas.

\section{Resultados e Discussão}

Identificaram-se 52 artigos em três das referidas bases de dados; apenas na SciELO o resultado foi "zero" (Fig. 1). Sete artigos foram eliminados por repetirem-se na EBSCO, BVS e PubMed. Efetuou-se a leitura dos títulos e resumos, dos 45 restantes.

Considerando o objetivo da pesquisa, foram eliminados 36 estudos, pelos seguintes motivos: (a) três estudos não se encontravam em livre acesso e não foi possível aceder aos mesmos, apesar de todos os esforços desenvolvidos para tal ${ }^{1}$; (b) 11 abordavam o rastreamento de deficiência em fetos/bebês; (c) seis tratavam de causas da deficiência auditiva em bebês; (d) dois aludiam ao conhecimento das gestantes sobre a surdez infantil; (e) três eram autorrelatos sobre o convívio com a deficiência; (f) cinco estudos foram realizados com gestantes com deficiência física e excluíram da amostra a deficiência sensorial; (g) na categoria denominada "outros" foram incluídos seis estudos, três deles focavam gestantes sem surdez e em três as participantes não eram gestantes.

Assim, apenas nove artigos foram elegíveis e incluídos neste estudo, os quais passaram por uma análise qualitativa. O fluxograma (Fig. 1) pormenoriza as etapas previstas pelos PRISMA e a partir dele é possível constatar que não foi aplicado nenhum critério de exclusão naqueles artigos elegíveis para leitura completa.

\footnotetext{
${ }^{1}$ Foram realizadas tentativas de contato tanto com bibliotecas de outras Universidades portuguesas, quanto diretamente com os autores via ResearchGate. Até a data de ressubmissão do artigo à revista, não foi possível aceder aos mesmos.
} 


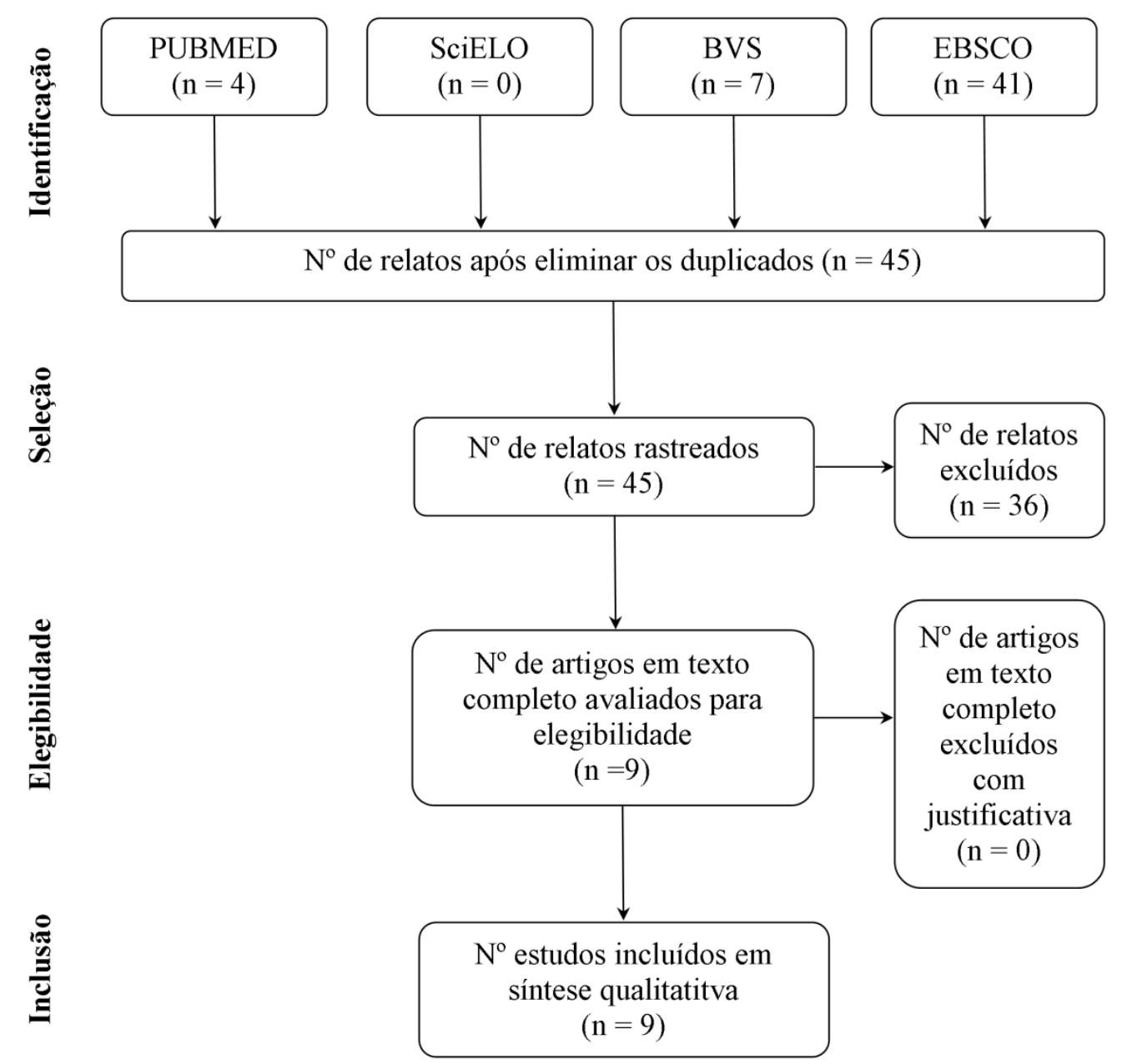

Figura 1. Estratégia de pesquisa baseada no PRISMA Statement (Moher et al., 2009)

A análise dos artigos incluídos permitiu a identificação de três categorias temáticas. Ainda que a amostra possa ser considerada pequena, os temas emergentes foram os seguintes: a deficiência/surdez e a exclusão; barreiras e preconceitos impostos às gestantes com deficiência/surdez e novas práxis precisam emergir.

\section{A deficiência/surdez e a exclusão}

Este estudo evidenciou que gestantes com deficiência/surdez vivenciam dois tipos de exclusão: uma velada e outra manifesta. A primeira constata-se pela ausência, na falta de dados censitários e até mesmo de estudos científicos sob o olhar da Psicologia. A exclusão manifesta evidencia-se no âmbito social e na (des)atenção à saúde física e mental destas mulheres.

A falta de precisão na quantificação de gestantes com deficiência conforme, pelo menos, ao tipo e ao nível é uma das constatações deste estudo, por exemplo, na Áustria, estima-se que $8,1 \%$ das mulheres em idade fértil convivem com prejuízos permanentes, 
porém não há registros da taxa de natalidade entre essas mulheres (Schildberger et al., 2017). No Reino Unido e nos Estados Unidos os dados referem-se ao número de gestantes com alguma deficiência, sem especificar quantas seriam surdas (Schildberger et al., 2017).

Semelhante aos dados identificados nesta amostra, em Portugal, em 2018 o número de nascimentos foi de 87.020 (PORDATA, 2019). Conforme o Censo de 2011, existiam 339 mulheres com idade entre 15 e 44 anos, que se autodeclararam como alguém que "não consegue efectuar a ação de ouvir" (Instituto Nacional de Estatística, 2019). No entanto, não há informações a respeito de gestantes/mães com algum tipo de deficiência ou disfuncionalidade, nestas mesmas bases de dados.

Nos Estados Unidos, passados trinta anos da promulgação da lei dos Americanos Portadores de Deficiência e trinta e três anos da existência do Sistema de Monitoramento de Avaliação de Riscos na Gravidez, somente em 2019 foram incluídas questões sobre deficiência no inventário a ser aplicado no país (D’Angelo et al., 2020). Houve um avanço significativo, ainda que tardio, no que tange às informações que serão coletadas a partir deste instrumento. Um passo importante fora dado, pois conhecer o universo e as necessidades desta população permitirá pensar em estratégias que minimizem suas dificuldades de acesso aos serviços de saúde.

Ademais à falta de estudos censitários, há uma carência, também, de estudos acadêmicos e a título de curiosidade científica, realizou-se outra busca, nas referidas bases, na mesma data, sem uso de filtros, com os seguintes termos MeSH/Descritores: "pregnant women" AND "Psychology" e os resultados foram bastante diferentes da busca anterior, conforme é possível constatar na Tabela 1. A tabela demonstra que a gestação é um tema bastante estudado pela Psicologia e, permite inferir que há muito a ser explorado pela mesma quando se trata de gestantes surdas.

\section{Tabela 1}

Comparativo de Resultados em Função do Uso da Palavra-chave "Deaf*"

\begin{tabular}{lcc}
\hline Base de dados & $\begin{array}{c}\text { Pregnant Women AND } \\
\text { Psychology AND Deaf* }\end{array}$ & $\begin{array}{c}\text { Pregnant Women AND } \\
\text { Psychology }\end{array}$ \\
\hline PubMed & 4 & 11260 \\
SciELO & 0 & 22 \\
EBSCO & 41 & 22378 \\
BVS & 7 & 10917 \\
Total & 52 & 44577 \\
\hline
\end{tabular}


Destaca-se, ainda, que os resultados obtidos com o primeiro grupo de palavras representam $0,11 \%$ dos resultados provenientes do segundo. Dentre estes estudos, pouco se abordou a respeito das variáveis psicológicas antes, durante e após a gestação. Dois mencionaram a autoeficácia e autoconsciência destas mulheres, que levaram adiante o desejo de ser mãe, mesmo com a falta de apoio familiar e profissional (Schildberger, Zenzmaier \& Koning-Bachmann, 2017; Wołowicz-Ruszkowska, 2016). Walsh-Gallagher, Sinclair e Mc Conkey (2012), por seu turno, abordaram sobre a representação da gestação/maternidade para estas mulheres, como uma afirmação da feminilidade, para além da realização de um desejo.

A invisibilidade revelada pela falta de dados censitários e de estudos científicos soma-se à exclusão manifesta. As gestantes surdas vivem uma exclusão social a qual é evidenciada pelo baixo status econômico e educacional, e pela ocupação de cargos com menores remunerações; razões pelas quais são também excluídas de informações pertinentes aos seus cuidados de saúde e aos do bebê (Akasreku, Habib \& Ankomah, 2018; Jackson, 2011; Mitra, Lu \& Diop, 2012; Mitra et al., 2015; Schildberger et al., 2017). Alguns autores apontam que a exclusão social somada à falta de suporte percebido dos profissionais de saúde, explicariam a procura tardia pelas consultas prénatais (Jackson, 2011; Mitra et al., 2012; Mitra et al., 2015; Walsh-Gallagher et al., 2012).

Referente à saúde das gestantes e de seus bebês, constata-se a importância dos cuidados pré-natais que incluem uma atenção voltada às condições físicas e mentais. A falta de informações e de suporte social são fatores associados ao aumento de ansiedade e depressão, durante a gestação (Almeida \& Arrais, 2016). No entanto, esta revisão não encontrou relato de apoio psicológico às gestantes surdas durante ou após esse período. O’Hearn (2006) também não encontrou estudos sobre esses cuidados com gestantes surdas.

A falta de estudos acadêmicos e censitários dificulta a elaboração de políticas públicas, de campanhas de promoção de saúde e prevenção de complicações durante e após a gestação (D’Angelo et al., 2020; Mitra et al., 2012; Mitra et al., 2015; WołowiczRuszkowska, 2016). Tal invisibilidade pode reforçar preconceitos e discriminações que estas mulheres enfrentam na sociedade e até mesmo nos serviços de saúde (Akasreku et al., 2018; Jackson, 2011; Schildberger et al., 2017; Walsh-Gallagher et al., 2012, Walsh-Gallagher et al., 2013; Wołowicz-Ruszkowska, 2016). 
O número de estudos sobre a saúde geral da população surda é sensivelmente superior às investigações sobre gestantes surdas/com deficiência. No entanto, as considerações são semelhantes a respeito da necessidade de ampliar investigações científicas e censitárias (Barnett et al., 2011; Druel et al., 2018; McKee et al., 2011a; McKee et al., 2011b; Woodcock \& Pole, 2007). Considera-se que o desconhecimento da realidade de todas e de cada uma, de suas limitações e potencialidades, fazem com que o preconceito e a rotulação ganhem espaço entre a gestante com deficiência/surda e o contexto ao qual ela está inserida.

\section{Barreiras e preconceitos impostos às gestantes com deficiência/surdez}

Barreiras físicas e atitudinais estão presentes no cotidiano de gestantes com deficiência/surdez (Mitra et. al. 2012). Há falta de acessibilidade aos prédios e às instalações, profissionais de saúde não se sentem preparados e/ou não recebem treinamento para lidar com pessoas com deficiência (Walsh-Gallager et al., 2013). Outros estudos também constataram que estes profissionais reconhecem a falta de formação e suas dificuldades para comunicarem-se com pessoas surdas (Gichane et al., 2017; Marquete et al., 2018; McKee et al., 2011b; Reis \& Santos, 2019; Tedesco \& Junges, 2013). No entanto, apesar de admitir a falta de conhecimento e habilidade para comunicar, os profissionais de saúde, não demonstraram atitude para sanar tal déficit (Marquete et al., 2018).

A barreira na comunicação, para além de comprometer a relação entre profissionais de saúde e gestantes, pode criar constrangimentos a curto, médio e longo prazo, uma vez que os cuidados pré-natais são importantíssimos para a saúde da mãe e do bebê. Ao realizar grupos de pré-natal psicológico com gestantes Wechsler et al. (2016) destacam a importância do preparo e conscientização destes profissionais no acompanhamento pré-natal. As autoras recomendam especial atenção àquelas gestantes com baixa escolaridade. Poderia-se inferir que esta recomendação seria adequada também para o acompanhamento de gestantes com deficiência/surdez.

A falta de suporte dos profissionais de saúde pode levar a uma procura tardia pelas consultas pré-natais (Jackson, 2011; Mitra et al., 2012; Mitra et al., 2015; WalshGallagher, et al., 2012). Gestantes com deficiência/surdez relatam falta de apoio à decisão de engravidar (Schildberger et al., 2017; Walsh-Gallagher et al., 2012; WalshGallager et al., 2013). Relatam, também, carência de informações/orientações ou apoio, 
dos médicos ou parteiras, sobre questões importantes para os seus cuidados e bem estar (Walsh-Gallager et al., 2012).

Uma pesquisa realizada na África do Sul, com 42 gestantes surdas, constatou que $93 \%$ delas receberam cuidados pré-natais e apenas $49 \%$ destas buscaram o serviço no primeiro trimestre da gestação, evidenciando a necessidade de ampliar a atenção dispensada a essas mulheres (Gichane et al., 2017). Outro estudo nos Estados Unidos constatou que as gestantes surdas demonstram menos satisfação do que as gestantes ouvintes com a comunicação médica e também com os cuidados gerais recebidos (O’Hearn, 2006).

$\mathrm{Na}$ sociedade e, em alguns casos nos serviços de saúde, a gestação entre mulheres com deficiência não é aceita e a qualidade da maternidade destas tende a ser questionada (Akasreku et al., 2018; Schildberger et al., 2017; Wołowicz-Ruszkowska, 2016). São julgadas por decidirem engravidar e manter a gestação (Akasreku et al., 2018; D’Angelo et al., 2020; Mitra et al., 2012; Mitra et al., 2015; Schildberger et al., 2017; Walsh-Gallagher et al., 2012; Walsh-Gallager et al., 2013; WołowiczRuszkowska, 2016).

Participantes de dois estudos relataram práticas discriminatórias por parte dos profissionais de saúde: médicos que as orientavam a abortar ou então entregar o filho para adoção (Schildberger et al., 2017; Walsh-Gallagher et al., 2012). Há também relatos de atendimento pré-natal inadequado, por parte destas mulheres (Mitra et al., 2012; Schildberger et al., 2017; Wołowicz-Ruszkowska, 2016).

O Quadro 1 exemplifica alguns depoimentos de gestantes com deficiência, que revelaram a descrença de familiares e profissionais de saúde nas suas habilidades/competências para serem mães, sentem-se, portanto, discriminadas por suas condições (Akasreku et al., 2018; Schildberger et al., 2017; Walsh-Gallagher et al., 2012; Walsh-Gallager et al., 2013; Wołowicz-Ruszkowska, 2016). WołowiczRuszkowska (2016) é assertiva ao afirmar que a maternidade não deve ser definida a partir da deficiência física ou sensorial, pois é um relacionamento pautado por emoções e responsabilidades. 


\section{Quadro 1}

Depoimentos de Gestantes com Deficiência Extraídos dos Estudos Revistos

\begin{tabular}{|c|c|}
\hline Estudos & Depoimentos \\
\hline Akasreku et al. (2018, p. 5) & $\begin{array}{l}\text { "- Por que você não tem pena de si mesma? Você não sabe que a gravidez é destina- } \\
\text { da a corpos capazes?" }\end{array}$ \\
\hline Wołowicz-Ruszkowska (2016, p. 85) & $\begin{array}{l}\text { "- A médica disse que na opinião dela, mesmo que ninguém a pedisse, é um risco } \\
\text { desnecessário ter um filho aleijado." }\end{array}$ \\
\hline Walsh-Gallagher (2012, p. 160) & $\begin{array}{l}\text { "- Todos [profissionais de saúde] estavam se olhando e ninguém estava falando co- } \\
\text { migo (...) o lugar estava lotado. Havia pessoas por toda parte, no entanto, durante } \\
\text { toda minha gravidez esse era o meu momento mais solitário". }\end{array}$ \\
\hline Schildberger et al. $(2017$, p. 4) & $\begin{array}{l}\text { "- Minha mãe disse: '- Por que você fez isso para você mesma?’ (...) Todos estavam } \\
\text { felizes com a gravidez da minha irmã e comigo, ninguém.” }\end{array}$ \\
\hline Schildberger et al. $(2017$, p. 7$)$ & "- É difícil para uma mulher ler os lábios, enquanto está sofrendo". \\
\hline
\end{tabular}

Os depoimentos ilustrados no quadro acima revelam o estereótipo social de que uma mulher com deficiência não seria capaz de ter uma experiência materna positiva. Para $70,5 \%$ dos respondentes, num estudo realizado numa zona rural de Gana, estas mulheres não deveriam ter sentimentos ou desejos sexuais. Há ainda a crença de que uma mulher com deficiência poderia transmitir sua condição ao feto de uma gestante não deficiente (Akasreku et al., 2018).

Se, na comunidade o desconhecimento científico cede espaço às crenças, que por sua vez geram situações de estigma às gestantes com deficiência/surdez, poderia-se inferir que nos hospitais/maternidades estas mulheres estariam livres destes atos. No entanto, existem relatos de maus tratos e abusos nas maternidades. Para Guichane et al. (2017), atitudes de indiferença não são exclusivos de gestantes surdas, porém tentem a ser agravadas devido suas dificuldades de comunicação e também por sofrerem discriminação devido à sua condição.

Para além dos familiares, profissionais de saúde e comunidade, o Estado também adota uma postura de preconceito e desconfiança com estas mulheres. WalshGallager et al. (2012) mencionam ações de controle voltadas às capacidades destas mulheres desempenharem suas funções maternas. Nove das dezessete participantes desta pesquisa foram assistidas por profissionais da saúde e do Serviço Social após o parto e todas sentiram ameaça de que seus filhos poderiam ser levados à adoção (Walsh-Gallager et al., 2012). Se estas mulheres estavam em sofrimento, isso não foi alvo de averiguação pelos profissionais, as mesmas mencionaram que receberam pouco ou nenhum apoio destes (Walsh-Gallager et al., 2012). 
Desse modo, poder-se-ia inferir que há uma relação da invisibilidade da temática, explorada nesta revisão, com a (falta de) formação dos profissionais de saúde e o tratamento (in)adequado de gestantes com deficiência/surdez. Faz-se necessário ampliar estudos, discutí-los na comunidade acadêmica e levá-los ao conhecimento da sociedade. Urge a implementação de novas ações a fim de promover a saúde integral destas gestantes, de seus filhos e familiares.

\section{Novas práxis precisam emergir}

Os estudos incluídos nesta revisão evidenciaram a necessidade da emersão de novos olhares e formas de pensar/atuar com esta população. Dentre as ações necessárias está a formação adequada dos profissionais de saúde (Schildberger et al., 2017; WalshGallager et al., 2013), no caso das gestantes surdas seria importante o conhecimento, por parte dos profissionais, da língua de sinais/gestual (Jackson, 2011).

É preciso reconhecer as desigualdades em saúde e o desconhecimento a respeito da gestação e maternidade entre mulheres com deficiência/surdas para ajustar as práticas, conforme as necessidades destas (D’Angelo et. al., 2020; Jackson, 2011; Mitra et al., 2012; Mitra et al., 2015; Walsh-Gallager et al., 2012; Walsh-Gallager et al., 2013). De acordo com O’Hearn (2006) a adesão aos cuidados pré-natais está associado à satisfação com as consultas, à qual depende da qualidade da comunicação com o médico.

Numa perspectiva macro, Wołowicz-Ruszkowska (2016) defende que o Estado deve promover ações para que estas mulheres consigam desempenhar suas funções maternas. Existem ações voltadas ao suporte para famílias com filhos com deficiência, no entanto, quando os pais possuem algum tipo de limitação este apoio é falho e/ou até mesmo inexistente (Waslsh-Gallager et al., 2012; Wołowicz-Ruszkowska, 2016).

É preciso ampliar estudos voltados a esta temática, pois como destaca Wołowicz-Ruszkowska (2016), raramente a maternidade entre mulheres com deficiência tem sido tema de pesquisa. Para Mitra et al. $(2012,2015)$ é indispensável obter dados populacionais deste público e destacam ainda a importância de identificar necessidades específicas das gestantes conforme o tipo e nível da incapacidade.

Jackson (2011) sugere que a comunicação em língua de sinais/gestual seria o ideal para compreender e ser compreendida. No entanto, enquanto isso não é possível, a autora indica ações inclusivas e uma delas é envolver o(a) companheiro(a) da gestante surda nas consultas pré-natais. A autora menciona que $90 \%$ das gestantes surdas 
possuem companheiro(a) com esta mesma condição e recomenda, por exemplo, que durante a realização da ecografia o profissional dê o microfone para os pais segurarem, pois assim sentirão, através da vibração, os batimentos cardíacos do feto.

\section{Considerações finais}

Esta revisão integrativa demonstrou que as gestantes surdas precisam ser vistas, "ouvidas", orientadas e apoiadas pelos diferentes setores da sociedade: família, comunidade, profissionais de saúde e pesquisadores, em especial pelos psicólogos. Diante do número limitado de estudos incluídos na síntese qualitativa, foi possível depreender a dimensão da problemática existente, uma vez que estas mulheres não recebem a devida atenção, o que consequentemente poder-se-ia inferir tratar-se de um problema de saúde pública. Afinal, é a saúde de, pelo menos, duas pessoas que estão em risco por não receber as orientações e apoios necessários nesta fase do desenvolvimento humano.

É importante destacar que os estudos incluídos nesta revisão discutem a atenção (ou a falta dela) dada às gestantes com deficiência, por profissionais de saúde (e não só). Quanto a Psicologia, a mesma está cada vez mais próxima das gestantes e seus familiares, porém parece que não tem dedicado seu olhar e "escuta" às gestantes surdas ou com outra deficiência. É possível que existam trabalhos realizados e, talvez, não publicados, o que reforçaria a necessidade de aproximação entre as práticas e as investigações científicas. De qualquer forma, compreende-se que é urgente dar visibilidade a esta problemática, bem como apresentar soluções.

Uma ação necessária, portanto, é olhar para as gestantes com deficiência, pois antes de delinear ações e pensar em mudanças é preciso considerar (e talvez aceitar) que estas mulheres desejam e podem ser mães, salvo em função das limitações impostas pela deficiência, identificadas por avaliação profissional. Quanto à Psicologia, há um vasto campo de atuação voltado tanto à gestante surda (ou com deficiência física/sensorial), quanto aos seus familiares e profissionais de saúde.

Há campo para programas pré e pós natais como espaço de "escuta" dos sentimentos/pensamentos e até dos preconceitos que estas mulheres estão sofrendo. As famílias também precisam ser incluídas tanto como rede de apoio, quanto para refletir a respeito das limitações, mas também das potencialidades de uma gestante com 
incapacidade física e/ou sensorial. Com os profissionais de saúde poder-se-ia pensar em melhorar as estratégias de comunicação, debater sobre a cultura surda e sensibilizar para a eliminação de barreiras, as quais têm dificultado a relação destes com estas gestantes.

\section{Referências}

Almeida, N. M. de C., \& Arrais, A.da R. (2016). O pré-natal psicológico como programa de prevenção à depressão pós-parto. Psicologia: Ciência $e$ Profissão, 36(4), 847-863. doi:10.1590/1982-3703001382014

Anderson, M. L., Craig, K. S. W., \& Ziedonis, D. M. (2017). Deaf people's helpseeking following trauma: experiences with and recommendations for the Massachusetts Behavioral Healthcare System. Psychol Trauma, 9(2), 239-248. doi:10.1037/tra0000219

Arrais, A. da R., \& Araujo, T.C.C.F.de. (2016). Pré-natal psicológico: perspectivas para atuação do psicólogo em saúde materna no Brasil. Revista da SBPH, 19(1), 103-116. Recuperado de http://pepsic.bvsalud.org/scielo.php?script=sci_arttext\&pid=S1516$08582016000100007 \& \operatorname{lng}=\mathrm{pt} \& \operatorname{lng}=\mathrm{pt}$

Akasreku, B. Dela, Habib, H., \& Ankomah, A. (2018). Pregnancy in disability: community perceptions and personal experiences in a rural setting in Ghana. Journal of Pregnancy, 2018, 1-12. doi:10.1155/2018/8096839

Barnett, S.; Klein, J. D.; Pollard, R. Q.; Samar, V.; Schlehofer, D.; Starr, M.; Pearson, T. A. (2011). Community participatory research with Deaf Sign Language Users to identify health inequities. American Journal of Public Health, 101(12), 2235-2238 doi:10.2105/AJPH.2011.300308

Benincasa, M.; Freitas, V.B.de; Romagnolo, A. N., Januário, B.S., \& Heleno, M.G.V. (2019). O pré-natal psicológico como um modelo de assistência durante a gestação. Revista da SBPH,22(1), 238-257. Recuperado de http://pepsic.bvsalud.org/scielo.php?script=sci_arttext\&pid=S151608582019000100013\&lng=pt\&tlng=pt.

D’Angelo, D. V., Cernich, A., Harrison, L., Kortsmit, K., Thierry, J. A. M., Folger, S., \& Warner, L. (2020). Disability and pregnancy: a cross-federal agency collaboration to collect population-based data about experiences around the time of pregnancy. Journal of Women's Health, 29(3), 291-296. doi:10.1089/jwh.2020.8309

Druel, V., Hayet, H., Esman, L., Clavel, M., \& Rougé Bugat, M.E. (2018). Assessment of cancers' diagnostic stage in a Deaf community-survey about 4363 deaf patients recorded in French units. BMC Cancer, 18(1), 93. doi:10.1186/s12885-017-3972-3

Equy, V., Derore, A., Vassort, N., Mongourdin, B., \& Sergent, F. (2012). Évaluation des actions favorisantl'accessibilité aux soins des patientes enceintes sourdes. Journal de Gynécologie Obstétrique et Biologie de La Reproduction, 41(6), 561- 
565. doi:10.1016/j.jgyn.2012.04.017

Gichane, M. W., Heap, M., Fontes, M., London, L., Programme, H. R., \& Africa, S. (2017). "They must understand we are people": pregnancy and maternity service use among signing deaf women in Cape Town. Disabil Health, 10(3), 434-439. doi:10.1016/j.dhjo.2017.03.016

Instituto Nacional de Estatística (2019). Anuário Estatístico de Portugal 2018. Recuperado https://www.ine.pt/xportal/xmain?xpid=INE\&xpgid=ine_publicacoes\&PUBLICAC OESpub_boui $=381689773 \&$ PUBLICACOESmodo $=2$

Jackson, M. (2011). Deafness and antenatal care: understanding issues with access. British Journal of Midwifery, 19(5), 280-284. doi:10.12968/bjom.2011.19.5.280

Marquete, V. F., Costa, M. A. R., \& Teston, E. F. (2018). Communication with hearing impaired people from the perspective of health professionals. Revista Baiana de Enfermagem, 32, 1-9. doi:10.18471/rbe.v32.24055

Mckee, M. M., Barnett, S. L., Block, R. C., \& Pearson, T. A. (2011a). Impact of communication on preventive services among deaf American Sign Language users. Amercian Journal of Preventive Medicine, 41(1), 75-79. doi:10.1016/j.amepre.2011.03.004

Mckee, M., Schlehofer, D., Cuculick, J., Starr, M., Smith, S., \& Chin, N. P. (2011b). Perceptions of cardiovascular health in an undesrserved community of deaf adults using American Sign Language. DisabilHea, 4(3), 192-197. doi:10.1016/j.dhjo.2011.04.001

Mckee, M. M., Paasche-Orlow, M., Winters, P. C., Fiscella, K., Sen, A., Pearson, T., \& Arbor, A. (2015). Assessing health literacy in deaf American Sign Language users. Journal of Health Communication, 20(2), 92-100. doi:10.1080/10810730.2015.1066468

Mitra, M., Lu, E., \& Diop, H. (2012). Smoking among pregnant women with disabilities. Women's Health Issues, 22(2), e233-e239. doi:10.1016/j.whi.2011.11.003

Mitra, M., Clements, K. M., Zhang, J., Iezzoni, L. I., Smeltzer, S. C., \& Long-Bellil, L. M. (2015). Maternal characteristics, pregnancy complications, and adverse birth outcomes among women with disabilities. Medical Care, 53(12), 1027-1032. doi:10.1097/MLR.0000000000000427

Moher D, L.A, Tetzlaff, J.A.D.G., \& The PRISMA Group (2009). Preferred reporting items for systematic reviews and meta analyses: The PRISMA Statement. PLoS Med 6(7): e1000097. doi:10.1371/journal.pmed.1000097.t001

O'Hearn, A. (2006). Deaf women's experiences and satisfaction with prenatal care : a comparative study. Family Medicine Journal, 38(10), 712-716. Recuperado de https://fmch.bmj.com/ 
PORDATA (2019). Nados vivos de mães residentes em Portugal: total e fora do casamento. Recuperado de: https://www.pordata.pt/Portugal/Nados+vivos $+\mathrm{de}+\mathrm{m} \% \mathrm{c} 3 \% \mathrm{a} 3 \mathrm{es}+$ residentes $+\mathrm{em}+\mathrm{Por}$ tugal + total $+\mathrm{e}+$ fora + do + casamento- 14

Reis, V. de S. L., \& Santos, A. M. dos. (2019). Knowledge and experience of family health team professionals in providing healthcare for deaf people. Revista CEFAC, 2l(1), 1-8. doi:10.1590/1982-0216/20192115418

Santos, A. S., \& Portes, A. J. F. (2019). Perceptions of deaf subjects about communication in primary health care. Revista Latino-Americana de Enfermagem, 27. doi:10.1590/1518-8345.2612.3127

Schildberger, B., Zenzmaier, C., \& König-Bachmann, M. (2017). Experiences of Austrian mothers with mobility or sensory impairments during pregnancy, childbirth and the puerperium: a qualitative study. BMC Pregnancy and Childbirth, 17(1), 201. doi:10.1186/s 12884-017-1388-3

Tedesco, J. dos R., \& Junges, J. R. (2013). Challenges for receiving hearing-impaired individuals in primary healthcare services. Cadernos de Saúde Pública, 29(8), 16851689. doi:10.1590/0102-311X00166212

Walsh-Gallagher, D., Sinclair, M., \& Mc Conkey, R. (2012). The ambiguity of disabled women's experiences of pregnancy, childbirth and motherhood: a phenomenological understanding. Midwifery, 28(2), 156-162. doi:10.1016/j.midw.2011.01.003

Walsh-Gallagher, D., Mc Conkey, R., Sinclair, M., \& Clarke, R. (2013). Normalising birth for women with a disability: the challenges facing practitioners. Midwifery, 29(4), 294-299. doi:10.1016/j.midw.2011.10.007

Wechsler, A. M., Reis, K. P. dos, \& Ribeiro, B. D. (2016). Uma análise exploratória sobre fatores de risco para o ajustamento psicológico de gestantes. Psicologia Argumento, 34(86), doi:10.7213/psicol.argum.34.086.AO07

Wołowicz-Ruszkowska, A. (2016). How polish women with disabilities challenge the meaning of motherhood. Psychology of Women Quarterly, 40(1), 80-95. doi:10.1177/0361684315600390

Woodcock, K., \& Pole, J. D. (2007). Health profile of deaf Canadians: analysis of the Cananda community health survey. Canadian Family Physician, 53(december), 2140-2140e7. Recuperado de https://www.cfp.ca/content/53/12/2140

Submetido em: 14/05/2020

Aprovado em: 03/08/2020 